\title{
Deserves a hearing? A case report of remitting tinnitus with $\mathrm{N}$-acetyl cysteine
}

The known pharmacokinetic profile of a medication does not always point to its therapeutic potential. Many treatments were discovered serendipitously as a consequence of careful clinical observation, often in the context of treatment of apparently unrelated conditions. Consequently, many widely-used therapies have current uses well removed from their initial indications, for instance the use of aspirin in cardiovascular protection..$^{1,2}$

In this vein, a 47-year-old woman enrolled in a randomised trial of the glutathione precursor, $\mathrm{N}$-acetyl cysteine (NAC; $2 \mathrm{~g}$ daily) for unipolar depression, noted a striking reduction in her pre-existing tinnitus. Consent was obtained from this patient for participation in this trial. The IRB of Deakin University confirmed that the manuscript falls within the scope of the trial data and therefore independent consent from the participant for this publication was not required. Her history was as follows: She presented with moderate to severe depression, scoring 26 on the Montgomery Asberg Depression Rating Scale (MADRS) at baseline, and had moderate anxiety, with a Hamilton Anxiety Scale (HAM-A) score of 21 . She smoked, did not drink alcohol, and had a past caesarean section, hysterectomy and cataract surgery. After the NAC trial, her MADRS and HAM-A scores both plummeted to 1 , corresponding to clinical remission, and her Quality of Life Enjoyment \& Satisfaction Questionnaire score increased from 54 to 72 .

Her tinnitus, for which no clear cause could be identified, commenced 6 years prior and it was attributed at the time to stress and hormonal problems. The tinnitus was described as being like a cricket making quite a noise. Before the trial, the noise was continuous and obvious, and she described that her head felt cloudy; she couldn't concentrate or relax and complained of feeling depressed and having a poor memory. All of these symptoms improved while on NAC treatment. Prior to treatment, she rated the severity of her tinnitus as "medium", but it was persistent and unending. After treatment, the tinnitus had largely disappeared, and only re-emerged when she reported being 'uptight' or 'stressed', but when she was relaxed the tinnitus was absent. Further, when the tinnitus did occur, the intensity was reduced and was, therefore, much less noticeable

Correspondence

Prof M Berk

email: mikebe@barwonhealth.org.au than it had been previously. She described the change as unbelievable.

Prompted by this observation it was intriguing to find that Neri et al described greater oxidative stress in elderly males with tinnitus than controls, and hypothesised that this may be related to endothelial damage, impairing the microcirculation of the labyrinth and acoustic and vestibular tracts. ${ }^{3}$ Interestingly, greater jugular than brachial differences in these markers were found in the tinnitus group. The same authors subsequently compared 44 patients and 25 healthy volunteers, and found greater oxidative stress in the tinnitus group. Soon after, Savastano et al described an open label study of 31 participants with unilateral idiopathic tinnitus administered phospholipids and vitamins (glycerophosphorylcholine,

glycerophosphorylethanolamine, beta-carotene, vitamin C, vitamin E). Treatment resulted in a reduction in subjective discomfort and tinnitus intensity. ${ }^{4}$

Glutathione is the brain's dominant free radical scavenger, and NAC promotes the endogenous production of glutathione by increasing cysteine. N-acetyl cysteine has an extensive track record including its use to counter paracetamol poisoning and treat schizophrenia and bipolar disorder ${ }^{1}$, but as yet there have been no published reports of its use in the treatment of tinnitus. That this finding occurred in the context of a study of unipolar depression minimises the likelihood that the report was the result of expectancy and a placebo effect. There is the potential that reductions in depressive symptoms may be related to the reported decrease in subjective tinnitus, especially given the presence of somatisation in depression. ${ }^{5}$ Similarly, we have postulated NAC's effects on depressive symptoms to be mediated by the overlapping mechanisms to those hypothesised to decrease tinnitus (reducing inflammation and oxidative stress). However, the participant reported decreased perceived noise, suggesting a direct impact on the tinnitus. Incidental findings such as this require replication, ideally in the context of placebo-controlled trials. However, given the paucity of treatment options and the chronic and disabling nature of the affliction, identification of potential novel treatments are of overt clinical and theoretical importance.

OM Dean 1,2,3, S Jeavons², GS Malhi',5, SM Cotton ${ }^{6,7}$, M Tanious ${ }^{4,5}$, K KohImann ${ }^{2}$, K Hewitt ${ }^{2}$, K Moss ${ }^{4}$, C Allwang ${ }^{8}$, I Schapkaitz², J Robbins ${ }^{2}$, H Cobb4, S Dodd ${ }^{1,3}$, A Bush², M Berk'1,2,3,6,7

${ }^{1}$ Deakin University, School of Medicine, Barwon Health, 
Geelong, Australia

2The Florey Institute of Neuroscience and Mental Health,

Parkville, Australia

3University of Melbourne, Department of Psychiatry,

Parkville, Australia

${ }^{4}$ Discipline of Psychiatry, Sydney Medical School, University of

Sydney, Sydney, Australia

${ }^{5}$ CADE Clinic, Department of Psychiatry, Royal North Shore

Hospital, St. Leonards, Australia

GOrygen Youth Health Research Centre, Parkville, Australia

${ }^{7}$ Centre of Youth Mental Health, University of Melbourne,

Parkville, Australia

8Department of Psychosomatic Medicine and Psychotherapy,

Klinikum rechts der Isar der TU, München, Germany

\section{References}

1. Dean O, Giorlando F, Berk M. N-acetylcysteine in psychiatry: current therapeutic evidence and potential mechanisms of action.J Psychiatry Neurosci 2011; 36:78-86.

2. Dean OM, Bush AI, Berk M. Translating the rosetta stone of $N$ acetylcysteine. Biol Psychiatry 2012; 71:935-6.

3. Neri S, Mauceri B, Cilio D et al. Tinnitus and oxidative stress in a selected series of elderly patients. Arch Gerontol Geriatr Suppl 2002; 8:219-23.

4. Savastano M, Brescia G, Marioni G. Antioxidant therapy in idiopathic tinnitus: preliminary outcomes. Arch Med Res 2007; 38:456-9.

5. Langguth B, Kleinjung T, Landgrebe M. Severe tinnitus and depressive symptoms: a complex interaction. Otolaryngol Head Neck Surg 2011; 145:519. 